\title{
Gaugeon formalism for perturbative quantum gravity
}

\author{
Sudhaker Upadhyay ${ }^{\mathrm{a}}$ \\ S. N. Bose National Centre for Basic Sciences, Block JD, Sector III, Salt Lake, Kolkata 700098, India
}

Received: 25 October 2013 / Accepted: 3 January 2014 / Published online: 1 February 2014

(C) The Author(s) 2014. This article is published with open access at Springerlink.com

\begin{abstract}
In this paper we investigate the Yokoyama gaugeon formalism for perturbative quantum gravity in a general curved spacetime. Within the gaugeon formalism, we extend the configuration space by introducing vector gaugeon fields describing a quantum gauge degree of freedom. Such an extended theory of perturbative gravity admits quantum gauge transformations leading to a natural shift in the gauge parameter. Further we impose the Gupta-Bleuler type subsidiary condition to remove the unphysical gaugeon modes. To replace the Gupta-Bleuler type condition by a more acceptable Kugo-Ojima type subsidiary condition we analyze the BRST symmetric gaugeon formalism. Further, the physical Hilbert space is constructed for the perturbative quantum gravity which remains invariant under both the BRST symmetry and the quantum gauge transformations.
\end{abstract}

\section{Introduction}

The usual perturbative approach of covariant quantum gravity in curved spacetime starts with the Einstein-Hilbert theory and expands the full Riemannian metric around a constant background. The diffeomorphism invariance then translates into a gauge symmetry of the fluctuation [1]. Consequently, the problem of formulating the corresponding quantum field theory in general curved spacetime is conceptually not much different from Yang-Mills theory. The study of quantum field theory, particularly, in de Sitter spacetime is very important as it gets relevance in inflationary cosmologies [2-5]. Recent observations indicate that our universe is expanding in such a rate that it may approach de Sitter spacetime asymptotically [6]. By the gauge invariant perturbative quantum gravity in curved space one has attempted in a great effort to unify gravity with Maxwell theory [7]. The gauge invariant gravity models have their relevance in string theories also [8-10].

\footnotetext{
a sudhakerupadhyay@gmail.com; sudhaker@boson.bose.res.in
}

Recently, significant developments have been made in the subject of quantum gravity in various directions [11-34].

On the other hand, the covariant quantization of perturbative gravity in general curved spacetime cannot be done without getting rid of the redundant degrees of freedom as the classical theory is gauge invariant [35]. The spurious degrees of freedom in the theory of perturbative gravity are removed by imposing a covariant gauge condition [35]. The gauge conditions are incorporated at the quantum level of the theory by adding suitable gauge-fixing and ghost terms to the classical action, which remains invariant under the fermionic rigid BRST transformation [14,36,37]. However, in the standard quantization of gauge theories, one does not consider the gauge transformation at the quantum level as there is no quantum gauge freedom. The quantum theory is defined only after fixing the gauge. Hayakawa and Yokoyama have shown that a shift in gauge parameter occurs through renormalization which affects the gauge-fixing condition [38].

Yokoyama's gaugeon formalism [39-45] provides a wider framework to quantize the gauge theories in which we discuss the quantum gauge transformation. The shift of the gauge parameter through renormalization has been naturally derived from the gauge structure within this formalism [39]. In this formalism, we extend the configuration space by introducing a set of extra fields (so-called gaugeon fields) in the effective Lagrangian density describing the quantum gauge freedom. It is obvious that the gaugeon modes do not contribute to physical processes and therefore one needs to remove them. First of all, Yokoyama achieved this by putting the Gupta-Bleuler type constraint on the gaugeon field, which has its own limitation [39]. Further, by introducing the BRST symmetric gaugeon formulation this situation is improved [46,47], which is facilitated by a more acceptable Kugo-Ojima type restriction [48,49]. The gaugeon formalism has been studied many times for the gauge theories in flat space time $[25,46,47,50-54]$; however, it has not been discussed in the context of gauge theories in curved spacetime. This provides a motivation to extend such a formalism for the 
quantum theory of gravity in curved spacetime. We show that this formalism also holds for the theory of linearized gravity where the fluctuations of the metric are treated as the gauge field.

In this paper, we consider the theory of perturbative quantum gravity in general curved metric space to discuss both the gauge and the BRST invariance. Further, to analyze the quantum gauge freedom of the theory, we extend the effective action by introducing two vector gaugeon fields. Within the gaugeon formalism, we investigate the quantum gauge transformation under which such an extended Lagrangian density (also called the Yokoyama Lagrangian density) remains form invariant. The transformed fields under a quantum gauge transformation satisfy the same equations of motion as the original ones, but with a shifted gauge parameter. Furthermore, we implement two subsidiary conditions, of KugoOjima type and Gupta-Bluler type, to remove the unphysical graviton and gaugeon modes, respectively. After that we demonstrate the BRST symmetric gaugeon formalism for perturbative gravity theory by further introducing ghost fields corresponding to the gaugeon fields. Such a BRST symmetric gaugeon action possesses both the BRST symmetry and the quantum gauge transformations. Further we show that by virtue of the BRST symmetry both the Kugo-Ojima type and the Gupta-Bluler type subsidiary conditions get converted into a single Kugo-Ojima type condition. Finally, the physical Hilbert space is constructed for the quantum gravity, which is annihilated by the BRST charge and also remains invariant under quantum gauge transformations.

We organize the paper as follows. In Sect. 2, we present the perturbative quantum gravity in general curved spacetime having gauge and BRST invariance. Section 3 is devoted to the study of the standard gaugeon formalism for perturbative quantum gravity. In Sect 4, the BRST symmetric gaugeon formalism is discussed. In the last section, we summarize our work.

\section{The perturbative quantum gravity in curved spacetime}

In this section, we analyze the BRST symmetry of perturbative quantum gravity in general curved spacetime. For this purpose, we begin with the Lagrangian density for the theory of classical gravity in general curved spacetime defined by

$\mathcal{L}_{c}=\sqrt{-\tilde{g}}(R-2 \Lambda)$,

where $\tilde{g}, R$, and $\Lambda$ are the determinant of the full metric $\tilde{g}_{a b}$, the Ricci scalar curvature, and the cosmological constant, respectively. Here units are adopted such that $16 \pi G=1$. The Lagrangian density remains invariant under the following infinitesimal transformation originating from its general coordinate invariance: $\delta_{\rho} \tilde{g}_{a b}=\nabla_{a} \rho_{b}+\nabla_{b} \rho_{a}$,

where $\nabla_{a}$ denotes the background covariant derivative and $\rho_{a}$ represents a vector field. In perturbative quantum gravity, one writes the full metric in terms of a fixed background metric and small perturbations around it. Therefore, we decompose the full metric $\tilde{g}_{a b}$ of classical gravity as

$\tilde{g}_{a b}=g_{a b}+h_{a b}$,

where $g_{a b}$ refers to the fixed background metric and $h_{a b}$ refers to small perturbations around the fixed metric. With the help of the above decomposition one can express the Lagrangian density for perturbative quantum gravity (1) in terms of the fluctuation $h_{a b}$. However, after being decomposed the transformation of $\tilde{g}_{a b}$ mentioned in (2) will be attributed to $h_{a b}$ as follows:

$\delta_{\rho} h_{a b}=\nabla_{a} \rho_{b}+\nabla_{b} \rho_{a}$.

The gauge invariance of the Lagrangian density (1) implies that there are redundancies in the physical degrees of freedom. These redundancies of the degrees of freedom give rise to constraints in the canonical quantization [55] and produce divergences in the generating functional in the path integral quantization. In order to remove these redundancies we need to break the local gauge covariance by fixing the gauge as follows [14]:

$G[h]_{a}=\left(\nabla^{b} h_{a b}-k \nabla_{a} h\right)=0$,

where $k \neq 1$ is the gauge parameter. For $k=1$ the conjugate momentum corresponding to $h_{00}$ vanishes and therefore the partition function becomes ambiguous. To avoid such ambiguities sometimes $k$ is written in terms of an arbitrary finite constant $\beta$ as follows: $k=(1+\beta) / \beta$ [35].

To incorporate the above gauge-fixing condition in the theory of linearized gravity at the quantum level we add the following covariant gauge-fixing term in the gauge invariant Lagrangian density of pure gravity:

$\mathcal{L}_{g f}=\sqrt{-g}\left[b^{a}\left(\nabla^{b} h_{a b}-k \nabla_{a} h\right)+\frac{\alpha}{2} b_{a} b^{a}\right]$,

where $\alpha$ is a gauge parameter and $b^{a}$ is a Nakanishi-Lautrup type auxiliary field.

Further, to compensate the contribution of the above gauge-fixing term in the functional integral we need to add the following Faddeev-Popov ghost term in the effective theory:

$$
\begin{aligned}
\mathcal{L}_{g h} & =\sqrt{-g} \bar{c}^{a} \nabla^{b}\left[\nabla_{a} c_{b}+\nabla_{b} c_{a}-2 k g_{a b} \nabla_{c} c^{c}\right] \\
& =\sqrt{-g} \bar{c}^{a} M_{a b} c^{b}
\end{aligned}
$$


where the Faddeev-Popov matrix operator $\left(M_{a b}\right)$ has the following form:

$$
M_{a b}=\nabla_{c}\left[\delta_{b}^{c} \nabla_{a}+g_{a b} \nabla^{c}-2 k \delta_{a}^{c} \nabla_{b}\right] .
$$

Here, we note that the Faddeev-Popov ghost $\left(c^{a}\right)$ and antighost $\left(\bar{c}^{a}\right)$ fields appearing in the theory of perturbative gravity are vector fields.

Now, the total effective Lagrangian density for perturbative quantum gravity in covariant gauge is defined by

$$
\mathcal{L}_{T}=\mathcal{L}_{c}+\mathcal{L}_{g f}+\mathcal{L}_{g h},
$$

which admits the following nilpotent BRST transformation:

$$
\begin{aligned}
& s h_{a b}=-\left(\nabla_{a} c_{b}+\nabla_{b} c_{a}\right), \\
& s c^{a}=-c_{b} \nabla^{b} c^{a}, \\
& s \bar{c}^{a}=b^{a}, s b^{a}=0 .
\end{aligned}
$$

We observe that the sum of the gauge-fixing and ghost parts of the effective Lagrangian density (i.e., $\mathcal{L}_{g f}+\mathcal{L}_{g h}=: \mathcal{L}_{g}$ ) is BRST exact, and with the help of the above BRST symmetry it can be expressed as [22]

$$
\begin{aligned}
\mathcal{L}_{g} & =s \sqrt{-g}\left[\bar{c}^{a}\left(\nabla^{b} h_{a b}-k \nabla_{a} h+\frac{\alpha}{2} b_{a}\right)\right], \\
& =s \Psi,
\end{aligned}
$$

where $\Psi$ denotes the gauge-fixing fermion of the theory with the following expression:

$\Psi=\sqrt{-g}\left[\bar{c}^{a}\left(\nabla^{b} h_{a b}-k \nabla_{a} h+\frac{\alpha}{2} b_{a}\right)\right]$.

In the next section, we will study the development of the quantum gauge transformation for the covariant linearized gravity theory using the standard gaugeon formalism.

\section{Yokoyama gaugeon formalism}

In this section, we analyze the quantum gauge transformations using the Yokoyama gaugeon formalism for perturbative quantum gravity in a general curved metric space. For this purpose, we construct the Yokoyama Lagrangian density for perturbative quantum gravity by incorporating the vector gaugeon fields $y^{a}$ and $y_{\star}^{a}$ satisfying Bose-Einstein statistics as follows:

$$
\begin{aligned}
\mathcal{L}_{y k} & =\mathcal{L}_{c}+\sqrt{-g} b^{a}\left(\nabla^{b} h_{a b}-k \nabla_{a} h\right)+\frac{\varepsilon}{2} \sqrt{-g}\left(y_{\star}^{a}+\lambda b^{a}\right)^{2} \\
& +\sqrt{-g} \bar{c}^{a} M_{a b} c^{b}+\sqrt{-g} \nabla^{b} y_{\star}^{a}\left[\nabla_{a} y_{b}\right. \\
& \left.+\nabla_{b} y_{a}-2 k g_{a b} \nabla_{c} y^{c}\right],
\end{aligned}
$$

where $\varepsilon$ is a sign factor $(= \pm 1)$ and $\lambda$ is the gauge parameter, which is identified with $\alpha$ of (6) as $\alpha=\varepsilon \lambda^{2}$.
The Lagrangian density (13) admits the quantum gauge transformation which enables us to vary the gauge parameter. The quantum gauge transformation is given by

$h_{a b} \rightarrow \hat{h}_{a b}=h_{a b}-\tau\left(\nabla_{a} y_{b}+\nabla_{b} y_{a}\right)$,

$y_{\star}^{a} \rightarrow \hat{y}_{\star}^{a}=y_{\star}^{a}-\tau b^{a}$,

$y_{a} \rightarrow \hat{y}_{a}=y_{a}$,

$b^{a} \rightarrow \hat{b}^{a}=b^{a}$,

$\bar{c}^{a} \rightarrow \hat{\bar{c}}^{a}=\bar{c}^{a}$,

$c^{a} \rightarrow \hat{c}^{a}=c^{a}$,

where $\tau$ is an infinitesimal transformation parameter of a bosonic nature. Under such a quantum gauge transformation the Lagrangian density (13) remains 'form invariant', i.e. it transforms as

$\mathcal{L}_{y k}(\hat{\phi}, \hat{\lambda})=\mathcal{L}_{y k}(\phi, \lambda)$,

where $\phi$ stands for all the fields collectively and $\hat{\lambda}$ is defined by

$\hat{\lambda}=\lambda+\tau$.

The form invariance implies that the quantum fields $\hat{\phi}$ and $\phi$ satisfy the same equations of motion with gauge parameters $\hat{\lambda}$ and $\lambda$, respectively.

Now, the BRST transformation for the Lagrangian density (13) is given by

$s h_{a b}=-\left(\nabla_{a} c_{b}+\nabla_{b} c_{a}\right)$,
$s c^{a}=-c_{b} \nabla^{b} c^{a}, \quad s \bar{c}^{a}=b^{a}$,
$s b^{a}=0, \quad s y^{a}=0, \quad s y_{\star}^{a}=0$.

Corresponding to the above BRST invariance, there exists a Noether current $J_{\mu}$ satisfying the conservation law

$\partial_{\mu} J^{\mu}=0$,

which has the nilpotent BRST charge $Q_{b}=\int d^{3} x \sqrt{-g} J^{0}$. To define the physical states, the unphysical gaugeon and graviton modes are removed by imposing the following two subsidiary conditions:

$Q_{b} \mid$ phys $\rangle=0$,

$y_{\star}^{a(+)}|\mathrm{phys}\rangle=0$,

where the first Kugo-Ojima type condition removes the unphysical gauge modes from the total Fock space and the second Gupta-Bleuler type condition removes the unphysical gaugeon modes. The second subsidiary condition makes sense when the field $y_{\star}^{a}$ satisfies the following free field equation:

$\nabla_{b} \nabla^{b} y_{\star}^{a}=0$, 
which is derived by using the equations of motion of the field $y^{a}$. Here the d'Alembertian is defined as $\nabla_{b} \nabla^{b}=$ $\frac{1}{\sqrt{-g}} \partial_{\mu}\left[\sqrt{-g} g^{a b} \partial_{b}\right]$. This free field equation guarantees the well-defined decomposition of the field $y_{\star}^{a}$ into positive and negative frequency parts. Therefore, for the second subsidiary condition it is mandatory for $y_{\star}^{a}$ to satisfy the free equation. However, for the Kugo-Ojima type condition based on the conserved charge one has no such kind of limitation.

\section{Gaugeon formalism with BRST symmetry}

In this section, we develop the BRST symmetric gaugeon formalism for perturbative quantum gravity where the two subsidiary conditions obtained in the previous section get replaced by a single Kugo-Ojima type subsidiary condition. With this motivation, we construct the BRST symmetric Yokoyama Lagrangian density as

$$
\begin{aligned}
\mathcal{L}_{y k b}= & \mathcal{L}_{c}+\sqrt{-g} b^{a}\left(\nabla^{b} h_{a b}-k \nabla_{a} h\right) \\
& +\frac{\varepsilon}{2}\left(y_{\star}^{a}+\lambda b^{a}\right)^{2}+\sqrt{-g} \bar{c}^{a} M_{a b} c^{b} \\
& +\sqrt{-g} \nabla^{b} y_{\star}^{a}\left[\nabla_{a} y_{b}+\nabla_{b} y_{a}-2 k g_{a b} \nabla_{c} y^{c}\right] \\
& +\sqrt{-g} K_{\star}^{a} M_{a b} K^{b},
\end{aligned}
$$

where $K_{\star}^{a}$ and $K^{a}$ are Faddeev-Popov ghosts corresponding to gaugeon fields $y_{\star}^{a}$ and $y^{a}$. The BRST transformation for the above Lagrangian density is given by

$$
\begin{aligned}
& s h_{a b}=-\left(\nabla_{a} c_{b}+\nabla_{b} c_{a}\right), \\
& s c^{a}=-c_{b} \nabla^{b} c^{a}, \quad s \bar{c}^{a}=b^{a}, \\
& s b^{a}=0, \quad s y^{a}=K^{a}, \quad s y_{\star}^{a}=0, \\
& s K_{\star}^{a}=y_{\star}^{a}, \quad s K^{a}=0 .
\end{aligned}
$$

It is easy to check the nilpotency (i.e. $s^{2}=0$ ) of the above BRST transformation. We further recast the Lagrangian density (21) with the help of the above BRST transformation, where the gauge-fixing and ghost parts are the BRST variation of the extended gauge-fixing fermion, as follows:

$$
\begin{aligned}
\mathcal{L}_{y k b} & =\mathcal{L}_{c}+s \sqrt{-g}\left[\bar{c}^{a}\left(\nabla^{b} h_{a b}-k \nabla_{a} h+\frac{\varepsilon \lambda}{2}\left(y_{a \star}+\lambda b_{a}\right)\right)\right. \\
& \left.-K_{\star}^{a}\left(M_{a b} y^{b}-\frac{\varepsilon}{2}\left(y_{a \star}+\lambda b_{a}\right)\right)\right] .
\end{aligned}
$$

Here the expression for the extended gauge-fixing fermion is given as

$$
\begin{aligned}
\Psi & =\sqrt{-g}\left[\bar{c}^{a}\left(\nabla^{b} h_{a b}-k \nabla_{a} h+\frac{\varepsilon \lambda}{2}\left(y_{a \star}+\lambda b_{a}\right)\right)\right. \\
& \left.-K_{\star}^{a}\left(M_{a b} y^{b}-\frac{\varepsilon}{2}\left(y_{a \star}+\lambda b_{a}\right)\right)\right] .
\end{aligned}
$$

This gauge-fixing fermion gets identified with the gaugefixing fermion given in Eq. (12) for vanishing gaugeon and corresponding ghost fields.
Now, the Noether charge $(Q)$ corresponding to the BRST symmetry transformation Eq. (22) annihilates the physical states of the total Hilbert space as follows:

$Q \mid$ phys $\rangle=0$,

which helps in defining the physical Hilbert space of the theory. This single subsidiary condition removes both the gaugeon modes and the unphysical graviton (gauge) modes from the physical subspace of states, as the BRST operator acts on both the gaugeon fields and the usual gauge fields. (For example, it can be seen from expression (22) that the gaugeon fields $y, y_{\star}, K$, and $K_{\star}$ form a BRST quartet which appears only as zero-normed states in the physical subspace [56].) Unlike the Gupta-Bleuler type condition, this single condition (25) does not have any kind of limitation.

Now, we establish the quantum gauge transformations under which the BRST invariant Yokoyama Lagrangian density (21) remains form invariant. These transformations are given by

$$
\begin{aligned}
& h_{a b} \rightarrow \hat{h}_{a b}=h_{a b}-\tau\left(\nabla_{a} y_{b}+\nabla_{b} y_{a}\right), \\
& y_{\star}^{a} \rightarrow \hat{y}_{\star}^{a}=y_{\star}^{a}-\tau b^{a}, \\
& y_{a} \rightarrow \hat{y}_{a}=y_{a}, \\
& b^{a} \rightarrow \hat{b}^{a}=b^{a}, \\
& \bar{c}^{a} \rightarrow \hat{\bar{c}}^{a}=\bar{c}^{a}, \\
& c^{a} \rightarrow \hat{c}^{a}=c^{a}+\tau K^{a}, \\
& K_{\star}^{a} \rightarrow \hat{K}_{\star}^{a}=K_{\star}^{a}-\tau \bar{c}^{a}, \\
& K^{a} \rightarrow \hat{K}^{a}=K^{a}, \\
& \lambda \rightarrow \hat{\lambda}=\lambda+\tau .
\end{aligned}
$$

It is straightforward to check that these transformations commute with the BRST transformation given in (22), which confirms that the BRST charge $Q$ remains unchanged under the above quantum gauge transformations:

$Q \rightarrow \hat{Q}=Q$,

where $\hat{Q}$ is the transformed BRST charge under the quantum gauge transformations. Therefore, the physical space of states $\mathcal{V}_{\text {phys }}$ annihilated by the charge $Q$ also remains intact under these transformations, i.e.

$\hat{\mathcal{V}}_{\text {phys }}=\mathcal{V}_{\text {phys }}$.

As a result the physical Hilbert space of quantum gravity $\mathcal{H}_{\text {phys }}=\mathcal{V}_{\text {phys }} / \operatorname{Im} Q$ is also invariant under both the BRST and the quantum gauge transformations.

\section{Conclusions}

In this paper, we have studied the BRST symmetry for perturbative quantum gravity in a general curved spacetime with 
a covariant gauge condition. Further, we have analyzed the Yokoyama gaugeon formalism for the theory of quantum gravity and discussed the quantum gauge degree of freedom. Within the analysis, we have constructed the Yokoyama Lagrangian density for the theory of quantum gravity by incorporating two vector gaugeon fields. The quantum gauge transformations have also been investigated under which the Yokoyama Lagrangian density for perturbative quantum gravity remains form invariant with a shift in the gauge parameter. It has been noticed that there exist unphysical modes also associated with both gaugeon and graviton fields and therefore one needs to remove them from the physical Hilbert space. We have removed them by imposing two subsidiary conditions, the Kugo-Ojima type and Gupta-Bleuler type. The Kugo-Ojima type subsidiary condition removes the unphysical gauge modes and the Gupta-Bleuler type condition removes the unphysical gaugeon modes. Moreover, for the Gupta-Bleuler type condition we have found a certain limitation.

Further, the BRST symmetric gaugeon formalism has been developed for the gravity theory which incorporates ghost fields also corresponding to gaugeon fields. The supremacy of the BRST version of the gaugeon formalism is that here Yokoyama's physical subsidiary condition of Gupta-Bleuler type translates into a more acceptable KugoOjima type condition. The BRST symmetric Yokoyama Lagrangian density possesses the quantum gauge transformation also, which commutes with the BRST symmetry of the theory. As a result, we have found that the physical state annihilated by the BRST charge is also invariant under quantum gauge transformations. Hence, a physical Hilbert space of perturbative quantum gravity has been constructed which remains invariant under quantum gauge transformations. We hope that such an analysis will be helpful in developing the full quantum theory of gravity. It will be interesting to generalize the quantum gauge transformations by making the bosonic transformation parameter field-dependent, which will lead to a field-dependent Jacobian (Upadhyay, in preparation). It will also be interesting to explore the gaugeon formulation in the framework of a generalized BRST transformation (Upadhyay, in preparation).

Open Access This article is distributed under the terms of the Creative Commons Attribution License which permits any use, distribution, and reproduction in any medium, provided the original author(s) and the source are credited.

Funded by $\mathrm{SCOAP}^{3}$ / License Version CC BY 4.0.

\section{References}

1. S. Weinberg, Gravitation and Cosmology. (John Wiley and Sons, New York, 1972)
2. S.W. Hawking, G.F. Ellis, The Large-Scale Structure of Space-Time (Cambridge University Press, Cambridge, 1973)

3. A.H. Guth, Phys. Rev. D 23, 347 (1981)

4. A.D. Linde, Phys. Lett. B 108, 389 (1982)

5. A. Albrecht, P.J. Steinhardt, Phys. Rev. Lett. 48, 1220 (1982)

6. S. Perlmutter, G. Aldering, G. Goldhaber, R.A. Knop, P. Nugent et al., Astrophys. J 517, 565 (1999)

7. A. Einstein, The Meaning of Relativity, 5th edn. (Princeton University Press, New Jersey, 1956)

8. A.H. Chamseddine, Int. J. Mod. Phys. A 16, 759 (2001)

9. T. Damour, S. Deser, J. McCarthy, Phys. Rev. D 47, 1541 (1993)

10. A.H. Chamseddine, Commun. Math. Phys. 218, 283 (2001)

11. C. Anastopoulos, B.L. Hu, J. Phys. Conf. Ser. 67, 012012 (2007)

12. M. Faizal, A. Higuchi, Phys. Rev. D 78, 067502 (2008)

13. I. Eyo Eyo III, Class. Quant. Grav. 25, 125002 (2008)

14. M. Faizal, Found. Phys. 41, 270 (2011)

15. M. Faizal, A. Higuchi, Phys. Rev. D 85, 12402 (2012)

16. M. Bojowald, AIP Conf. Proc. 910, 294 (2007)

17. A. Ashtekar, Nuovo Cim. B 122, 135 (2007)

18. J.-P. Wu, Y. Ma, Phys. Rev. D 86, 124044 (2012)

19. M. Faizal, JETP 114, 400 (2012)

20. M. Faizal, J. Phys. A 44, 402001 (2011)

21. D. Benedetti, S. Speziale, JHEP 1106, 107 (2011)

22. M. Faizal, Phys. Lett. B 705, 120 (2011)

23. K.-J. Hamada, Found. Phys. 39, 1356 (2009)

24. M. Faizal, Class. Quant. Grav. 29, 035007 (2012)

25. M. Faizal, Mod. Phys. Lett. A 27, 1250147 (2012)

26. R.B. Mann, M.B. Young, Class. Quant. Grav. 24, 951 (2007)

27. M. Faizal, Mod. Phys. Lett. A 27, 1250075 (2012)

28. M. Faizal, Class. Quant. Grav. 29, 215009 (2012)

29. M. Faizal. arXiv:1301.0224

30. K. Giesel, T. Thiemann, Class. Quant. Grav. 24, 2565 (2007)

31. M. Faizal. arXiv: 1303.5478

32. L. Modesto, Gen. Rel. Grav. 37, 191 (2005)

33. M. Faizal, Mod. Phys. Lett. A 28, 1350034 (2013)

34. Z. Bern, Living Rev. Relativ. 5, 5 (2002)

35. A. Higuchi, S.S. Kouris, Class. Quant. Grav. 18, 4317 (2001)

36. S. Upadhyay, Phys. Lett. B 723, 470 (2013)

37. S. Upadhyay, Ann. Phys. 340, 110 (2014)

38. M. Hayakawa, K. Yokoyama, Prog. Theor. Phys. 44, 533 (1970)

39. K. Yokoyama, Prog. Theor. Phys. 51, 1956 (1974)

40. K. Yokoyama, Prog. Theor. Phys. 59, 1699 (1978)

41. K. Yokoyama, Prog. Theor. Phys. 60, 1167 (1978)

42. K. Yokoyama, Phys. Lett. B 79, 79 (1978)

43. K. Yokoyama, R. Kubo, Prog. Theor. Phys. 52, 290 (1974)

44. K. Yokoyama, M. Takeda, M. Monda, Prog. Theor. Phys. 60, 927 (1978)

45. K. Yokoyama, M. Takeda, M. Monda, Prog. Theor. Phys. 64, 1412 (1980)

46. K. Izawa, Prog. Theor. Phys. 88, 759 (1992)

47. M. Koseki, M. Sato, R. Endo, Prog. Theor. Phys. 90, 1111 (1993)

48. T. Kugo, I. Ojima, Prog. Theor. Phys. Suppl No. 66, 1 (1979)

49. T. Kugo, I. Ojima, Nucl. Phys. B 144, 234 (1978)

50. M. Koseki, M. Sato, R. Endo, Bull. Yamagata Univ. Nat. Sci. 14, 15 (1996)

51. Y. Nakawaki, Prog. Theor. Phys. 98, 5 (1997)

52. R. Endo, M. Koseki, Prog. Theor. Phys. 103, 3 (2000)

53. H. Miura, R. Endo, Prog. Theor. Phys. 117, 4 (2007)

54. M. Faizal, Commun. Theor. Phys. 57, 637 (2012)

55. M. Henneaux, C. Teitelboim, Quantization of Gauge Systems (University Press, Princeton, 1992)

56. T. Kugo, I. Ojima, Phys. Lett. B 73, 459 (1978)

57. T. Kugo, I. Ojima, Prog. Theor. Phys. 60, 1869 (1978) 